\title{
Generation Gap for Screening and Treatment of Hepatitis C Virus in Saga Prefecture, Japan: An Administrative Database Study of 35,625 Subjects
}

\author{
Hiroshi Isoda ${ }^{1,2}$, Satoshi Oeda ${ }^{2}$, Ayako Takamori ${ }^{3}$, Keiko Sato ${ }^{3}$, Michiaki Okada ${ }^{1}$, \\ Shinji Iwane ${ }^{2}$, Hirokazu Takahashi ${ }^{1}$, Keizo Anzai ${ }^{1}$, Yuichiro Eguchi ${ }^{2}$ and Kazuma Fujimoto ${ }^{4}$
}

\begin{abstract}
:
Objective Despite recent advances in hepatitis C virus (HCV) treatment, some $\mathrm{HCV}$-positive individuals are unaware of their infection. The present study aimed to assess the rate and age distribution in Saga Prefecture regarding i) $\mathrm{HCV}$ infection and $\mathrm{HCV}$ screening and ii) direct acting anti-viral (DAA) treatment.

Methods HCV screening data collected at a medical institution or in the workplace were obtained from the administrative database in Saga Prefecture between April 1, 2008 and March 31, 2014. DAA treatment data from Saga Prefecture were obtained from the DAA treatment reimbursement recipient database between October 2014 and March 2017.

Results There were 35,625 individuals who underwent HCV screening, and the HCV positive rate was $1.18 \%$ (421 individuals), which increased in an age-related manner. The screening rate in the screened populations peaked at 45-74 years of age (approximately 6\%) and decreased in the younger and older generations. The estimated percentage of DAA treatment peaked at $65-74$ years old $(65.8 \%)$ and significantly decreased inversely with age in the younger generations; only $9.4 \%$ of HCV carriers received DAA treatment in the 20to 34-year age group. The proportion of subjects who received a complete physical examination for DAA treatment was higher in the subjects who were screened at a medical institution than in those screened at the workplace.

Conclusion The rate of subjects who underwent HCV screening and DAA treatment was not high, especially in the younger generation, in Saga Prefecture. This group should be targeted for HCV screening and treatment.
\end{abstract}

Key words: direct acting antiviral treatment, interferon, infection, HCV carrier

(Intern Med 59: 169-174, 2020)

(DOI: 10.2169/internalmedicine.3248-19)

\section{Introduction}

The estimated number of individuals who are chronically infected with hepatitis $\mathrm{C}$ virus (HCV) in Japan is between 1.5 and 2 million (approximately $1 \%$ of the total population), and HCV infection, in addition to hepatitis B virus infection, has been a major cause of hepatocellular carcinoma (HCC) (1-5). Whereas the prevalence of HCV infection has decreased in Japan in a time-dependent manner, HCV infec- tion and mortality caused by HCC and HCV-related diseases remain serious problems (1-5).

$\mathrm{HCV}$ treatment has dramatically improved in recent years, and the most recent HCV treatment breakthrough was associated with the development of direct-acting antivirals (DAAs) (6-9). The introduction of a combination of DAAs as fixed-dose formulations in 2014 dramatically changed the treatment of patients with HCV genotype $1 \mathrm{~b}(\mathrm{HCV}-1 \mathrm{~b})$ in Japan (9-11). Several phase III trials conducted in Japanese HCV-1b-infected patients indicated the potent efficacy

${ }^{1}$ Department of Internal Medicine, Faculty of Medicine, Saga University, Japan, ${ }^{2}$ Liver Center, Saga University Hospital, Japan, ${ }^{3}$ Division of Clinical Research Center, Saga University Hospital, Japan and ${ }^{4}$ International University of Health and Welfare, Faculty of Medicine, Japan Received: April 23, 2019; Accepted: July 24, 2019; Advance Publication by J-STAGE: September 18, 2019 Correspondence to Dr. Satoshi Oeda, ooeda-sag@umin.org 
(based on sustained viral responders) of different DAAs, which were associated with few adverse events (12-14). The development of DAAs has thus made HCV infection a treatable disease, with cure rates above $90 \%$ (15).

However, despite recent advances in $\mathrm{HCV}$ treatment, many HCV-positive individuals in Japan are unaware of their HCV infection and/or have limited access to the appropriate medical care $(16,17)$, suggesting that early HCV detection and treatment of new cases might be paramount for reducing the morbidity and mortality that are associated with HCV infection in Japan. Nationwide screening for HCV in Japan started in 2002 as part of the health examinations that are provided by the municipal governments, and the screening was performed at primary care medical institutions or in the workplace as part of routine occupational health checkups. The hepatitis medical care coordinators, beginning in Yamanashi Prefecture (18-20), facilitated HCV screening and treatment throughout Japan. Because of the high mortality rate of HCC in Saga Prefecture, HCV screening began in 1994 in Saga (18).

The purpose of the present study was to i) evaluate the number of subjects who received HCV screening in Saga Prefecture, ii) estimate the number of the infections using the participation and positive rate of HCV screening, and iii) determine the proportion of subjects who completed the intensive complete physical examination who were HCVpositive and received DAA treatment in Saga Prefecture.

\section{Materials and Methods}

The data were obtained from the administrative database that included HCV screening and treatment in Saga Prefecture, and the Saga Prefecture database and Saga University database between April 1, 2008, and March, 31, 2017, were pooled. The cost of $\mathrm{HCV}$ screening and $\mathrm{HCV}$ antibody blood tests was covered by the government of Saga Prefecture. Subjects underwent HCV screening at 268 medical institutions in Saga, or in the workplace as part of routine occupational health checkups.

Borderline subjects with intermediate or low HCV antibody titers underwent the HCV RNA test. Subjects with high HCV antibody titers or who were HCV RNA-positive were recommended to visit a medical institution for the intensive complete physical examination that is required for DAA treatment, which is covered by partial medical expense subsidies through the Japanese National Health Insurance program. They received DAA treatment with any of the following five combinations: i) daclatasvir + asunaprevir; ii) sofosbuvir + ledipasvir; iii) sofosbuvir + ribavirin; iv) ombitasvir + paritaprevir + ritonavir; or v) elbasvir + grazoprevir. Data on DAA treatment reimbursement recipients in Saga Prefecture between October 1, 2014, and March 31, 2017, were recorded in the liver disease database.

The protocol of the present study was approved by the Institutional Review Board of Saga University Hospital. All individuals who participated in HCV screening and received
DAA treatment reimbursement provided their written informed consent to participate in this study.

The subjects were analyzed by age as of 2014 and in 10year intervals. Subjects under 20 years of age who were not included in the HCV screening were excluded from the survey. Individuals 20 to 34 years of age were grouped together because of the limited number of such subjects. The age groups were as follows: 20-34 years, 35-44 years, 45-54 years, 55-64 years, 65-74 years, 75-84 years, and $\geq 85$ years. The estimated number of HCV-positive individuals in Saga Prefecture was obtained by multiplying the population by the HCV-positive rate. The populational data from Saga in 2013 published by Saga Prefecture were used in the present calculation. The proportion of individuals who underwent the intensive complete physical examination at a medical institution after receiving an $\mathrm{HCV}$-positive result was calculated in order to compare the implementation rate of the complete physical examination between screening at a medical institution and screening at the workplace.

Statistical analyses were conducted using a chi-square test to compare the distribution of the subjects. Statistical significance for the objectives was set at $\mathrm{p}<0.05$. All statistical analyses were performed using the JMP pro software program, ver. 13.1 (JMP Japan., Tokyo, Japan).

\section{Results}

\section{Number and distribution of subjects screened for HCV}

There were 35,625 subjects who underwent HCV screening in Saga Prefecture between April 2008 and November 2014. Among participants who underwent HCV screening, 16,928 were men, and 18,570 were women, and the sex was unknown for 127 participants.

There were $421 \mathrm{HCV}$-positive results (1.2\%). Table shows the number of subjects screened for $\mathrm{HCV}$ and the number of HCV-positive subjects. The age distribution was different between the overall population and the HCV-positive subjects $(\mathrm{p}<0.001)$. The proportion of HCV-positive individuals significantly increased in an age-related manner, from $0.5 \%$ in the 20 - to 34 -years age group to $3.6 \%$ in the $\geq 85$ years age group $(\mathrm{p}<0.001)$.

The estimated number of HCV-positive individuals in Saga in 2013 was 8,480. There were 2,541 people who underwent DAA treatment for HCV in Saga between October 2014 and March 2017. In addition, there was a significant difference in the distribution of the number of treated and untreated HCV carriers $(\mathrm{p}<0.001)$. The precise analysis of this distribution is shown in Fig. 1.

Fig. 2 shows the proportion of HCV-screened subjects in each age group in Saga Prefecture. The rate of subjects screened for HCV in each generation were 4.2\% (20-34 years), 5.5\% (35-44 years), 6.3\% (45-54 years), 6.3\% (55-64 years), $6.1 \%$ (65-74 years), $4.0 \%$ (75-84 years), and $2.9 \%$ ( $\geq$ 85 years). Among these generations, the percentage of sub- 
Table. Positive Rate of Anti-HCV Antibody and Estimated Number of HCV Carriers in Order of Age Group.

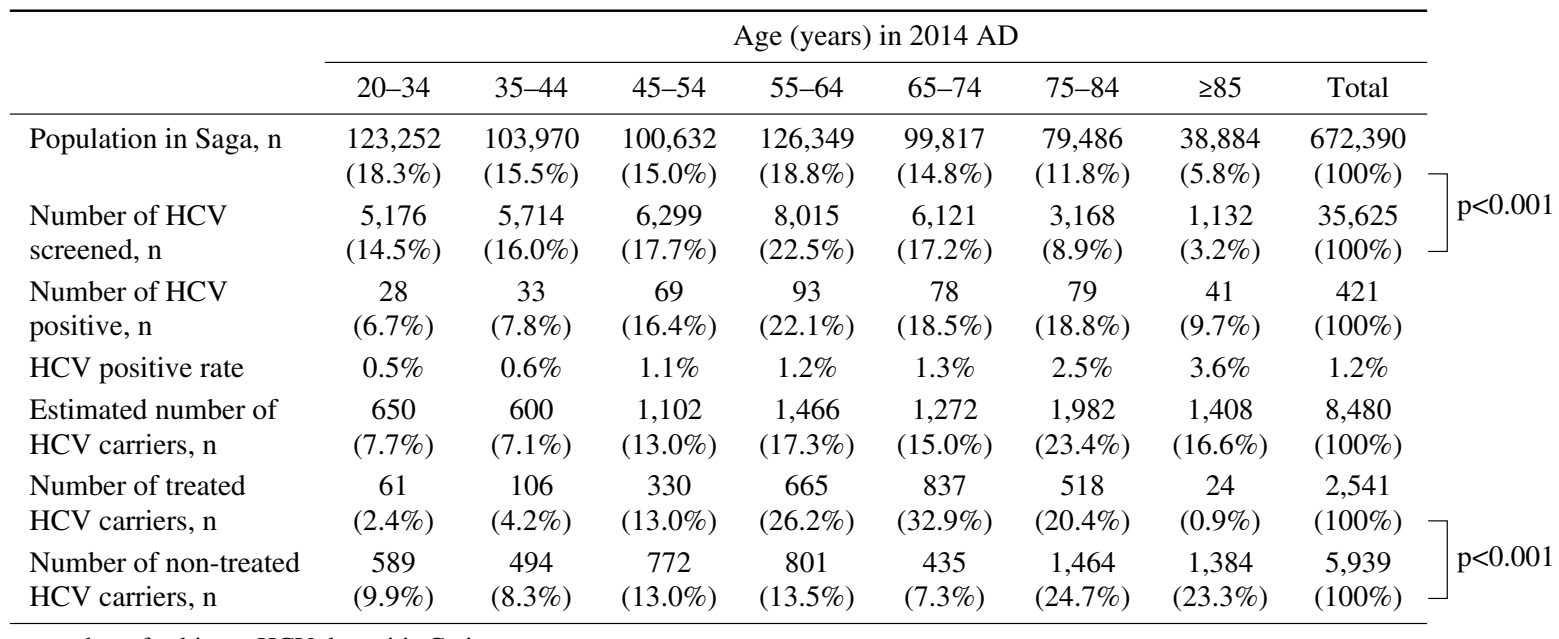

n: number of subjects, HCV: hepatitis $\mathrm{C}$ virus

Distribution between the Saga population and the number of HCV patients screened was statistically different $(\mathrm{p}<0.001)$. Distribution between treated and non-treated HCV carriers was statistically different $(\mathrm{p}<0.001)$.

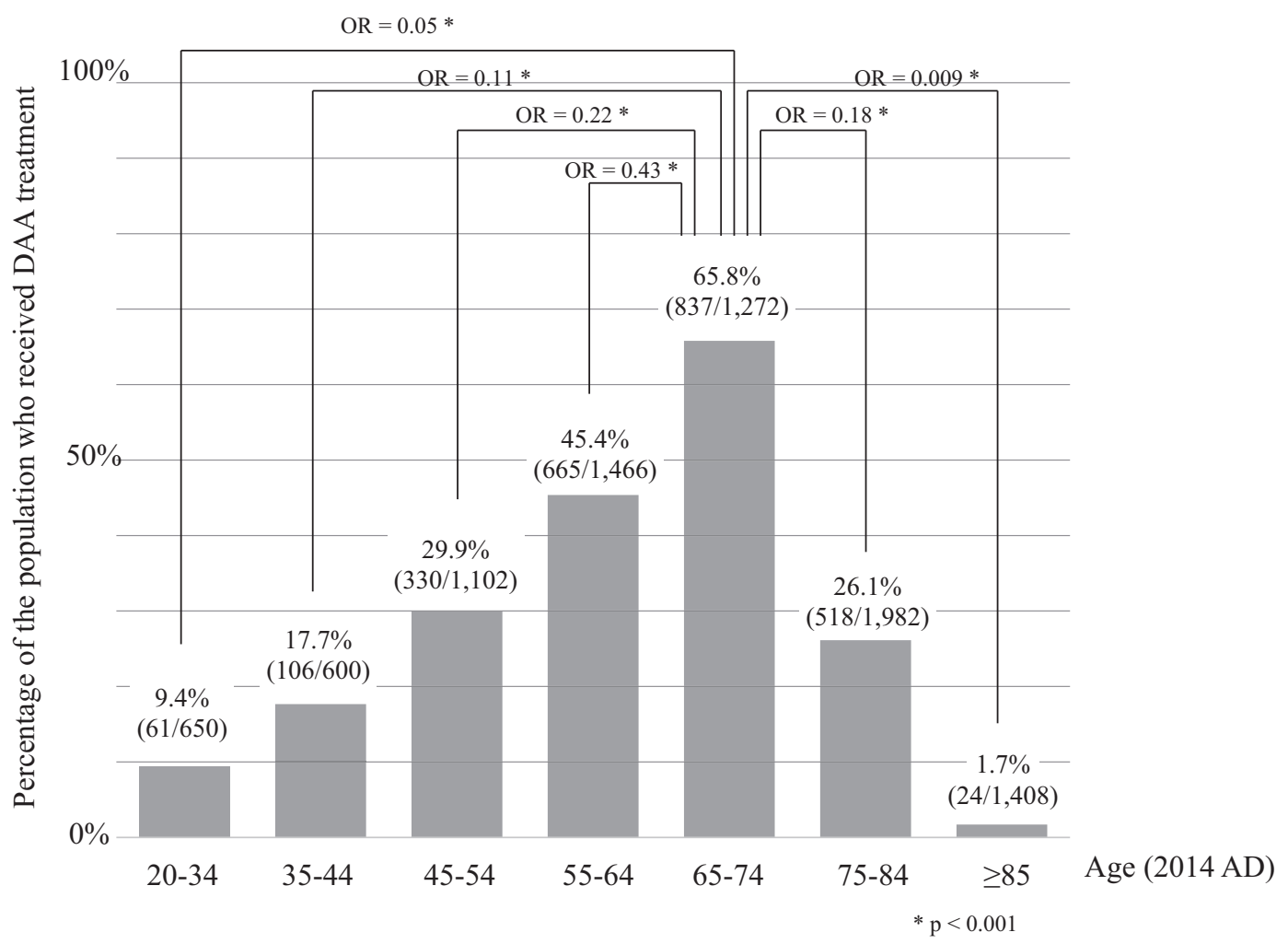

Figure 1. The proportion of patients who received DAA treatment between October 1, 2014, and March 31, 2017. The percentage was calculated from the estimated number of hepatitis $C$ virus carriers in Saga Prefecture. DAA: direct-acting antivirals, OR: odds ratio

jects who underwent HCV screening peaked among subjects who were 45-54 years old, 55-64 years old, and 65-74 years old, and the percentage who received HCV screening in the 65- to 74-years age group was significantly higher compared with other generations including the 20- to 34-years, 35- to 44 -years, 75 - to 84 -years, and $\geq 85$ years groups.

\section{Number and distribution of recipients who received DAA treatment}

Fig. 1 indicates the number of DAA treatment recipients by age group in 2014. Between October 2014 and March 2017, there were 2,541 DAA treatment recipients. There was an unequal distribution of recipients, with a peak of $65.8 \%$ in the 65- to 74-years group. The percentage of recipients 


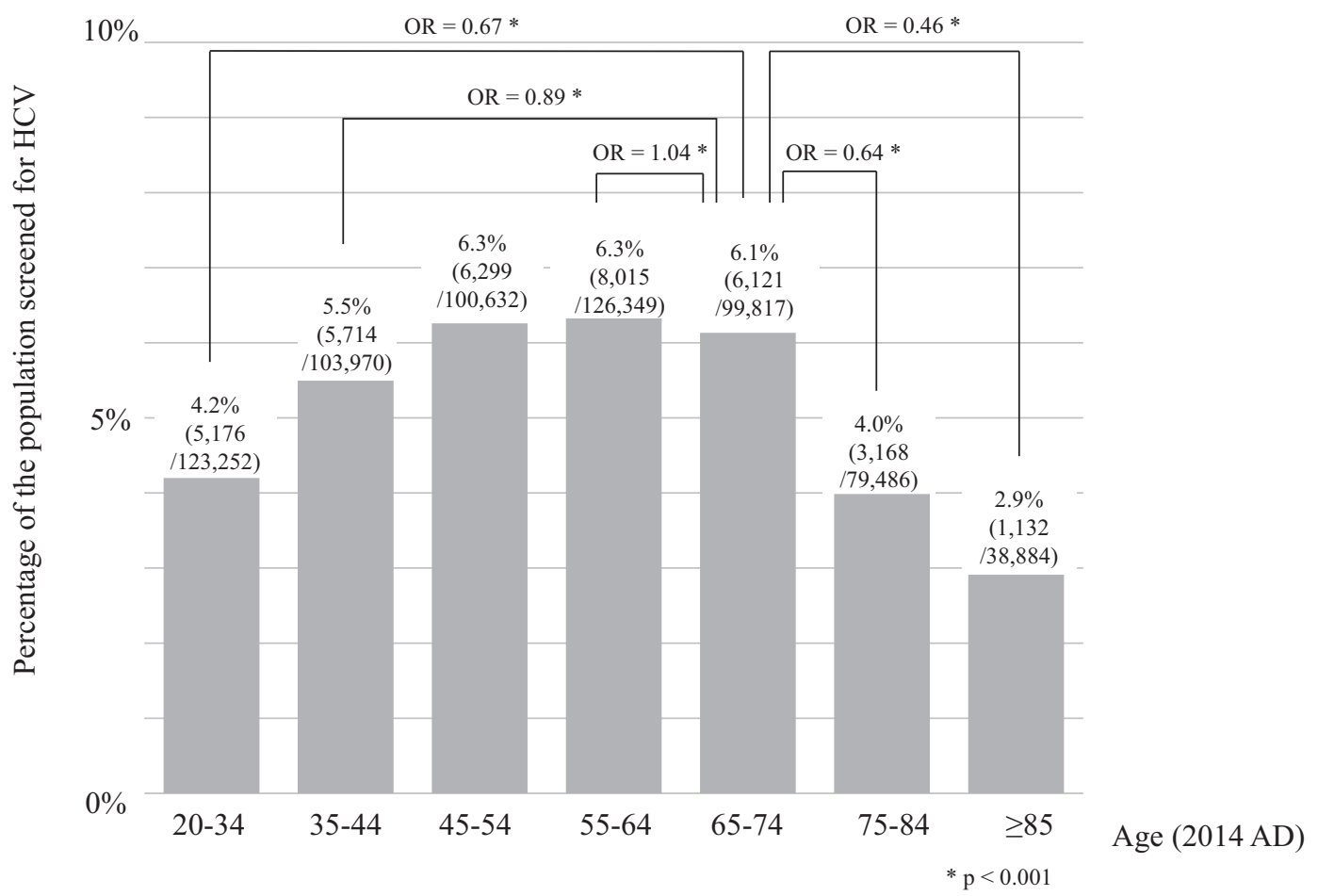

Figure 2. The proportion of subjects who were screened for hepatitis C virus (by each generation) in Saga Prefecture between April 2008 and November 2014. OR: odds ratio

who received DAA treatment decreased inversely with age in the age groups $<64$ years, and the percentage decreased in the 75 - to 84 -years and $\geq 85$ years groups compared with the 65- to 74-years group. The age-related distribution for who received DAA treatment was not affected by gender in the present study (data not shown).

\section{Proportion of the HCV-positive individuals who un- derwent a complete physical examination after HCV screening}

Between April 2013 and March 2016, HCV screening identified 271 individuals who were HCV-positive. Among the $271 \mathrm{HCV}$-positive subjects, 94 were identified in the workplace, and 177 were identified at a medical institution. Among the HCV-positive subjects, 142 (52.4\%) received a complete physical examination. Fig. 3 compares the proportion of HCV-positive individuals who underwent a complete physical examination after being identified in the workplace and those who were identified at the medical institution. Over $50 \%$ of the HCV-positive subjects who were identified at a medical institution received a complete physical examination $(65.5 \%)$, which was significantly higher than the percentage of $\mathrm{HCV}$-positive subjects who were identified in the workplace $(27.7 \%, \mathrm{p}<0.001)$. While the average age of subjects who underwent screening at a medical institution was significantly higher than those screened at the workplace $(56.2 \pm 16.8$ years old vs. $52.2 \pm 13.1$ years old, $\mathrm{p}<0.05)$ and the gender distribution was not markedly different between the two groups, age adjustment had no influence on the results of Fig. 3.

\section{Discussion}

The present study indicates that i) the percentage of subjects who received HCV screening in Saga Prefecture was relatively low, and ii) among the screened $\mathrm{HCV}$-positive subjects, the proportion of those who received DAA treatment was not high, especially in the younger generations in Saga Prefecture.

As indicated in the present study, the proportion of subjects who underwent HCV screening was not high in Saga Prefecture, and the HCV screening percentage was particularly low among the younger generations (20-44 years old) and older generation ( $\geq 75$ years old). The low screening rate in the younger generations might be because they are busy with work and/or have little interest in their own health. A previous study indicated that subjects who received $\mathrm{HCV}$ screening in the workplace lacked sufficient knowledge about hepatitis and HCV screening (21). Compared to the younger generations, the low HCV screening rate in the older generation might be a result of little interest in their own health, and HCV detection at a medical institution may have been because of a comorbidity before routine $\mathrm{HCV}$ screening.

Among HCV-positive subjects in Saga Prefecture, approximately $30 \%$ received DAA treatment, as indicated in Fig. 1. Among all generations, $65.8 \%$ of subjects who were 65-74 years old received DAA treatment, while the treatment rate was markedly decreased in the younger generations. Several reasons might explain the reduced DAA treatment rate in the younger generation, including being busy 


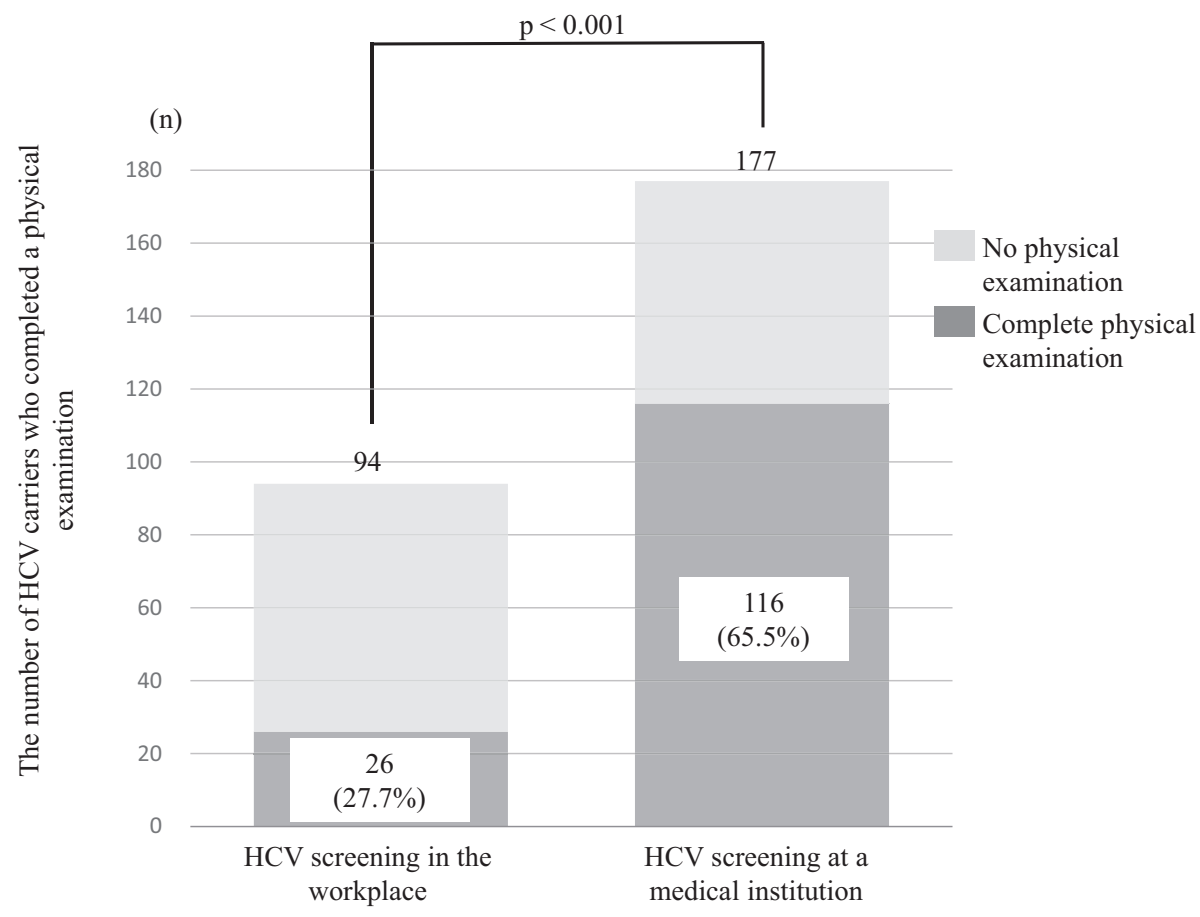

Figure 3. Rate of subjects who received a complete physical examination among the hepatitis C virus positive subjects screened between April 1, 2013, and March 31, 2016. Results are compared between screening at the workplace and in a medical institution.

with work, having little interest in HCV, the medical cost of the DAA treatment, and having a bad impression of painful HCV interferon treatment (22-24). In Japan, subjects 65-74 years of age, which is just after the retirement age limit, might have enough time and money for DAA treatment and be particularly interested in preserving their health. The low treatment rate in the older generations ( $\geq 75$ years old) might be because of several factors in addition to the potential reasons cited for the younger generations. In addition to a low sustained viral responder rate and many side effects of interferon therapy $(22,23)$, several physicians in Japan do not use this therapeutic approach for older HCV carriers (25).

The present study indicated that relatively few HCVpositive subjects who were identified in the workplace went on to receive a complete physical examination, which is required for DAA treatment, compared to HCV-positive subjects identified at a medical institution. Similar to the low $\mathrm{HCV}$ screening rate, being busy and having no interest in health care might be the main reasons for this low percentage. In addition, many individuals might have missed the notification, as the results of $\mathrm{HCV}$ screening performed at the workplace are mailed to the subject's home or corresponding workplace. The medical institution assessed the HCV-positive subjects more closely using information for DAA treatment of HCV. Based on these results, several projects have been proposed at the Ministry of Health, Labour and Welfare in Japan (26), and the Saga Prefecture government has started to recommend that HCV screening be included in the routine medical checkup at the workplace without any individual charge, resulting in a 10-fold increase in the HCV screening rate in the last year, which might in- crease the frequency of DAA treatment.

The present study did not include cases of HCV detected at hospital admission blood tests or a gynecological checkup. For HCV treatment, interferon-based therapy was not included. HCV treatment using DAAs has shown marked progress, and almost all patients have achieved $\mathrm{HCV}$ eradication without any serious side effects. The present findings suggest that many $\mathrm{HCV}$-positive individuals in the working-age population might not be receiving the appropriate treatment for $\mathrm{HCV}$. HCV screening and DAA treatment are insufficient in the younger and older generations in Japan. The early detection and treatment of HCV, especially in the younger generation, is important in order to prevent the progression of HCV-related diseases $(26,27)$. Therefore, further intensive HCV screening and treatment in Japan are warranted.

The authors state that they have no Conflict of Interest (COI).

\section{Acknowledgement}

This work was supported by Saga Prefecture. The authors would like to thank Mr. Yuuki Kamura, Mr. Daisuke Yoshihara, Mr. Shuichi Furukawa, and Ms. Aiko Ikegami from the Department of Health and Welfare in Saga Prefecture. We also thank Jodi Smith, $\mathrm{PhD}$, for editing a draft of this manuscript.

\section{References}

1. Tanaka J, Akita T, Ohisa M, et al. Trends in the total numbers of HBV and HCV carriers in Japan from 2000 to 2011. J Viral Hepatol 25: 363-372, 2018.

2. Uchida S, Satake M, Kurisu A, et al. Incidence rates of hepatitis C 
virus infection among blood donors in Japan: a nationwide retrospective cohort study. Transfusion 58: 2880-2885, 2018.

3. Sugiyama A, Fujii T, Nagashima S, et al. Pilot study for hepatitis virus screening among employees as an effective approach to encourage employees who screened positive to receive medical care in Japan. Hepatol Res 48: E291-E302, 2018.

4. Tanaka J, Koyama T, Mizui M, et al. Total numbers of undiagnosed carriers of hepatitis C and B viruses in Japan estimated by age- and area-specific prevalence on the national scale. Intervirology 54: 185-195, 2011.

5. Omata M, Kanda T, Wei L, et al. APASL consensus statements and recommendations for hepatitis $\mathrm{C}$ prevention, epidemiology, and laboratory testing. Hepatol Int 10: 681-701, 2016.

6. Omata M, Kanda T, Wei L, et al. APASL consensus statements and recommendation on treatment of hepatitis C. Hepatol Int 10: 702-726, 2016

7. Ji F, Wei B, Yeo YH, et al. Systematic review with meta-analysis: effectiveness and tolerability of interferon-free direct-acting antiviral regimens for chronic hepatitis $\mathrm{C}$ genotype 1 in routine clinical practice in Asia. Aliment Pharmacol Ther 47: 550-562, 2018.

8. Suda G, Ogawa K, Morikawa K, Sakamoto N. Treatment of hepatitis C in special populations. J Gastroenterol 53: 591-605, 2018.

9. Ogawa E, Furusyo N, Nomura $\mathrm{H}$, et al. NS5A resistanceassociated variants undermine the effectiveness of ledipasvir and sofosbuvir for cirrhotic patients infected with HCV genotype $1 \mathrm{~b}$. J Gastroenterol 52: 845-854, 2017

10. Ogawa E, Furusyo N, Yamashita N, et al. Effectiveness and safety of daclatasvir plus asunaprevir for patients with hepatitis $\mathrm{C}$ virus genotype $1 \mathrm{~b}$ aged 75 years and over with or without cirrhosis. Hepatol Res 47: E120-E131, 2017.

11. Tamori A, Enomoto M, Kawada N. Recent advances in antiviral therapy for chronic hepatitis C. Mediators Inflamm 2016: 6841628, 2016.

12. Swallow E, Kelley C, Signorovitch J, et al. Daclatasvir + asunaprevir versus sofosbuvir/ledipasvir for hepatitis C genotype 1 in Japanese patients: an indirect comparison. J Comp Eff Res 5: 273-279, 2016.

13. Mizokami M, Yokosuka O, Takehara T, et al. Ledipasvir and sofosbuvir fixed-dose combination with and without ribavirin for 12 weeks in treatment-naive and previously treated Japanese patients with genotype 1 hepatitis $\mathrm{C}$ : an open-label, randomised, phase 3 trial. Lancet Infect Dis 15: 645-653, 2015.

14. Itokawa N, Atsukawa $M$, Tsubota $A$, et al. Efficacy of directacting antiviral treatment in patients with compensated liver cirrhosis: a multicenter study. Hepatol Res 49: 125-135, 2019.

15. Kawakubo M, Eguchi Y, Okada M, et al. Chronic hepatitis C treatment with daclatasvir plus asunaprevir does not lead to a decreased quality of life. Intern Med 57: 1959-1966, 2018.
16. Tanaka J, Akita T, Ohisa M, et al. Trends in the total numbers of HBV and HCV carriers in Japan from 2000 to 2011. J Viral Hepat 25: 363-372, 2018.

17. Obora A, Kojima T, Hamaguchi M. The transition of participants who received health check-up programs and had a positive result of viral hepatitis B or C, but didn't consult a hospital, for the last two decades. Kanzo 57: 213-219, 2016 (in Japanese, Abstract in English).

18. Iwane S, Oeda S, Eguchi Y. Reducing the mortality rate of hepatitis and liver cancer in Japan. J Gen Fam Med 18: 205-211, 2017.

19. Kanzaki N, Iwane S, Oeda S, et al. Categorization and characterization of activities designed to help health-care professionals involved in hepatitis care increase their awareness of the disease: the classification of hepatitis medical care coordinators. Intern Med 58: 1825-1834, 2019.

20. Maekawa S, Enomoto N. Viral factors influencing the response to the combination therapy of peginterferon plus ribavirin in chronic hepatitis C. J Gastroenterol 44: 1009-1015, 2009.

21. Sugiyama A, Fujii T, Nagashima S, et al. Pilot study for hepatitis virus screening among employees as an effective approach to encourage employees who screened positive to receive medical care in Japan. Hepatol Res 48: E291-E302, 2018.

22. Kainuma M, Furusyo N, Kajiwara E, et al. Pegylated interferon $\alpha$ $2 \mathrm{~b}$ plus ribavirin for older patients with chronic hepatitis $\mathrm{C}$. World J Gastroenterol 16: 4400-4409, 2010.

23. Arase Y, Suzuki F, Suzuki Y, et al. Side effects of combination therapy of peginterferon and ribavirin for chronic hepatitis-C. Intern Med 46: 1827-1832, 2007.

24. Ito $\mathrm{Y}$, Sakata $\mathrm{Y}$, Yoshida H, et al. High cost of hospitalization for colonic diverticular bleeding depended on repeated bleeding and blood transfusion: analysis with diagnosis procedure combination data in Japan. Digestion 96: 76-80, 2017.

25. Nagao Y, Kawakami Y, Yoshiyama T, Sata M. Analysis of factors interfering with the acceptance of interferon therapy by HCVinfected patients. Med Sci Monit 14: PI45-PI52, 2008.

26. Oza N, Isoda H, Ono T, Kanto T. Current activities and future directions of comprehensive hepatitis control measures in Japan: the supportive role of the Hepatitis Information Center in building a solid foundation. Hepatol Res 47: 487-496, 2017.

27. Suda G, Ogawa K, Morikawa K, Sakamoto N. Treatment of hepatitis C in special populations. J Gastroenterol 535: 591-605, 2018.

The Internal Medicine is an Open Access journal distributed under the Creative Commons Attribution-NonCommercial-NoDerivatives 4.0 International License. To view the details of this license, please visit (https://creativecommons.org/licenses/ by-nc-nd/4.0/).

(C) 2020 The Japanese Society of Internal Medicine Intern Med 59: 169-174, 2020 